\title{
Improved high-contrast imaging with on-axis telescopes using a multistage vortex coronagraph
}

\author{
Dimitri Mawet, ${ }^{1, *}$ Eugene Serabyn, ${ }^{1}$ J. Kent Wallace, ${ }^{1}$ and Laurent Pueyo ${ }^{1,2,3}$ \\ ${ }^{1}$ Jet Propulsion Laboratory, California Institute of Technology, 4800 Oak Grove Drive, Pasadena, California 91109, USA \\ ${ }^{2}$ Space Telescope Science Institute, 3700 San Martin Drive, Baltimore, Maryland 21218, USA \\ ${ }^{3} J o h n s$ Hopkins University, Physics and Astronomy, 366 Bloomberg Center, \\ 3400 North Charles Street, Baltimore, Maryland 21218, USA \\ *Corresponding author: dmawet@eso.org
}

Received December 16, 2010; revised March 3, 2011; accepted March 3, 2011; posted March 22, 2011 (Doc. ID 139775); published April 15, 2011

\begin{abstract}
The vortex coronagraph is one of the most promising coronagraphs for high-contrast imaging because of its simplicity, small inner working angle, high throughput, and clear off-axis discovery space. However, as with most coronagraphs, centrally obscured on-axis telescopes degrade contrast. Based on the remarkable ability of vortex coronagraphs to move light between the interior and exterior of pupils, we propose a method based on multiple vortices, that without sacrificing throughput, reduces the residual light leakage to $(a / A)^{n}$, with $n \geq 4$, and $a$ and $A$ being the radii of the central obscuration and primary mirror, respectively. This method thus enables high contrasts to be reached even with an on-axis telescope. (C) 2011 Optical Society of America

OCIS codes: $\quad 070.6110,110.6770,350.1260$.
\end{abstract}

Coronagraphy enhances contrast in astrophysical scenes where the goal is imaging faint features near bright objects. The typical example is an extrasolar planet located less than an arcsecond from its much brighter host star. The vortex coronagraph (VC) [1-4] can observe very close to bright stars, and so it is one of the most promising of the coronagraph types. The vector vortex coronagraph (VVC), which is a realization of the VC based on a circularly symmetric half-wave plate [5], has been extensively demonstrated in the lab, in visible [6] and near IR [7] light. Two VVC masks have been installed on the $200 \mathrm{in}$. Hale telescope at Palomar, and have been used behind an off-axis unobscured subaperture corrected with adaptive optics to successfully image brown dwarfs and extrasolar planets $[\underline{7}, \underline{8}]$. However, as with any coronagraph, additional diffraction due to the secondary mirror obstruction present in on-axis telescopes will degrade achievable contrast. Here we show that this extra leakage can be greatly reduced by using two VC stages in sequence.

A VC applies a helical phase of the form $e^{i \phi}$, with $\phi=l \theta$, where $\theta$ is the focal plane azimuthal coordinate and $l$ is the (even) vortex topological charge, to a telescope's focal plane field, by means of a transparent phase mask (Fig. 1). The light then propagates downstream to a pupil image (the Lyot plane), where for an ideal unobscured circular input pupil (Fig. 1, left), all of the (on-axis) starlight appears outside of the geometric image of the input pupil, rendering the interior of the output pupil empty of the incoming on-axis illumination (Fig. 1, right). A Lyot-plane aperture then blocks all of the diffracted starlight. Light from the off-axis objects misses the center of the vortex and propagates normally.

The effect of a charge $l=2$ vortex phase, $e^{i 2 \theta}$, applied to the ideal focal plane field (Airy pattern), $\frac{2 J_{1}(k \rho A)}{k \rho A}$, of a filled circular aperture of radius $A$, where $k$ is the wavenumber and $\rho$ is the radial coordinate in the focal plane, has been calculated analytically $[1,3]$, and the Fourier transform of $e^{i 2 \theta} \times \frac{2 J_{1}(k \rho A)}{k \rho A}$ to the pupil (Lyot) plane yields

$$
E_{\text {Lyot }}(r, \psi)=\left\{\begin{array}{cc}
0 & r<A \\
e^{i 2 \psi}\left(\frac{A}{r}\right)^{2} & r>A
\end{array} .\right.
$$

The field outside the pupil area thus has a $1 / r^{2}$ falloff in this case.

Including now a central obstruction of radius $a$, an on-axis telescope has a pupil field distribution given by

$$
E_{\text {pup }}(r, \psi)= \begin{cases}0 & r<a \\ 1 & a<r<A \\ 0 & r>A\end{cases}
$$

Using the superposition principle, a centrally obscured pupil can be seen as the difference between a filled pupil of radius $A$ and a smaller filled pupil of radius $a$ (Fig. $\underline{2}$, A), with the field distribution

$$
E_{\text {pup }}(r, \psi)=\left\{\begin{array}{cc}
-1 & r<a \\
0 & r>a
\end{array}\right\}+\left\{\begin{array}{cc}
1 & r<A \\
0 & r>A
\end{array}\right\}
$$

Applying the same process to each component yields

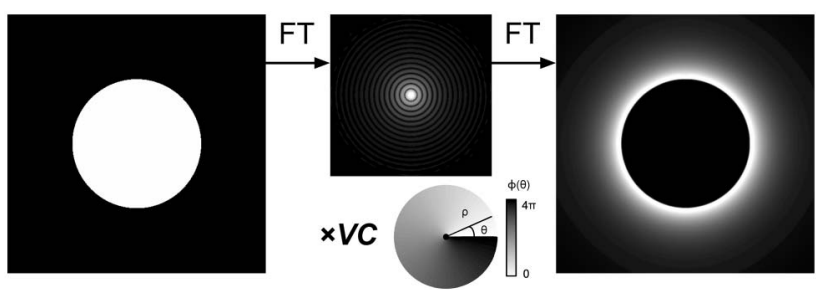

Fig. 1. Illustration of the diffraction effect of the vortex phase mask on a filled aperture (left). All of the on-axis coherent light appears outside of the geometric image of the input pupil (right). A circular aperture (Lyot stop) then blocks it all. FT, Fourier transform. 


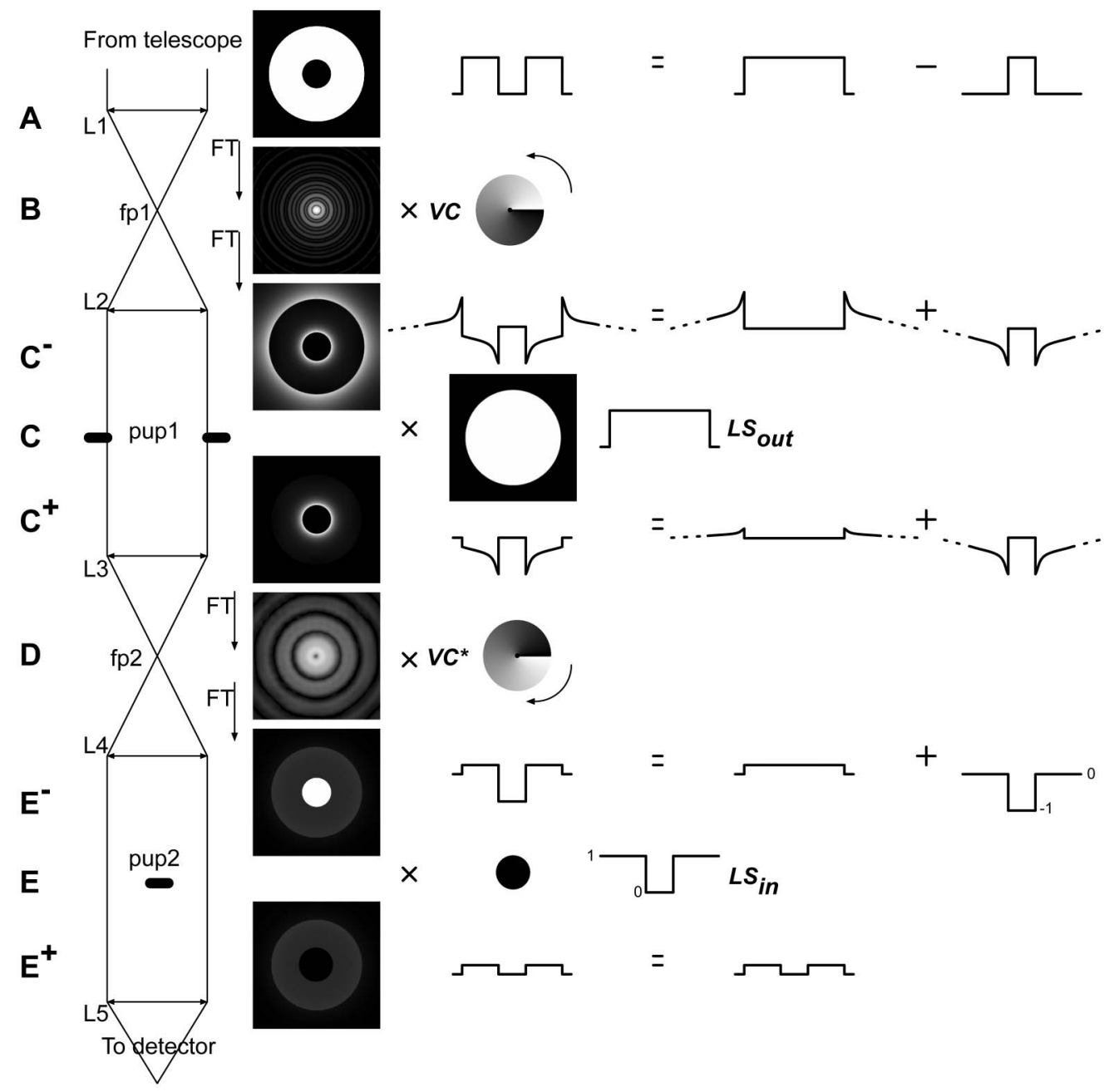

Fig. 2. Layout of the two-stage VC. A. Obscured entrance pupil decomposed into the difference of two filled apertures with radii $A$ and $a<A$. B. Application of the VC phase ramp $e^{i \phi}$ to the image produced by the imaging lens L1. $\mathbf{C}^{-}$. The pupil plane distribution after pupil imaging lens L2. The crosscuts show the linear decomposition of the field into the sum of a positive term $(A / r)^{2}$ for the filled pupil of radius $A$, and another negative term $-(a / r)^{2}$ corresponding to the filled pupil of radius $a<A$. C. First Lyot stop $L S_{\text {out }}$. $\mathbf{C}^{+}$. Post-Lyot stop field. The crosscuts show the decomposition of the residual field as the sum of the negative pupil term $-(a / r)^{2}$, and a second term that leads to cancellation beyond $A$. D. Image in the second focal plane after L3; note that the broad distribution now arises predominantly from the effect of $a$. Also shown is the conjugated vortex $\mathrm{VC}^{*}$, with a phase ramp $e^{-i \phi}$. $\mathbf{E}^{-}$. Pupil plane field after the second vortex, decomposed into the sum of an outer pupil of radius $A$ with a reduced amplitude $(a / A)^{2}$, and the inner pupil of radius $a$ of amplitude -1 . E. Application of the second, inner Lyot stop $\left(L S_{\text {in }}\right) . \mathbf{E}^{+}$. The final exit pupil after the second stage is a replica of the original entrance pupil left with an intensity reduced by the factor $(a / A)^{4}$.

$$
E_{\text {pup } 1}(r, \psi)=\left\{\begin{array}{ll}
0 & r<a \\
-e^{i 2 \psi}\left(\frac{a}{r}\right)^{2} & a<r<A \\
e^{i 2 \psi}\left[\left(\frac{A}{r}\right)^{2}-\left(\frac{a}{r}\right)^{2}\right] & r>A
\end{array} .\right.
$$

The field beyond radius $A$ can be removed by the opaque Lyot stop (Fig. 2, C), leaving the post Lyot stop field

$$
E_{\text {Lyot1 }}(r, \psi)= \begin{cases}0 & r<a \\ -e^{i 2 \psi}\left(\frac{a}{r}\right)^{2} & a<r<A . \\ 0 & r>A\end{cases}
$$

The residual field interior to the pupil (between $a$ and $A$ ) leads to contrast degradation in the subsequent focal plane image, as the fraction of the total energy remaining inside the pupil is $(a / A)^{2}$, or 0.01 for a $10 \%$ central obscuration.
We now show that a pair of conjugated VCs in series reduces the light leakage due to the central obscuration by an additional factor of $(a / A)^{2}$, leading to a total leakage of $(a / A)^{4}$. Our approach is based on the remarkable ability of the vortex mask to move the light between the interior and exterior of the pupil (Eq. (1)). Using superposition and the self-similarity of the $1 / r^{2}$ terms, the field after the first Lyot stop can be rewritten (Fig. $\underline{2}, \mathbf{C}^{+}$)

$$
E_{\mathrm{Lyot1}}(r, \psi)=\left\{\begin{array}{ll}
0 & r<a \\
-e^{i 2 \psi}\left(\frac{a}{r}\right)^{2} & a<r<A \\
e^{i 2 \psi}\left(\frac{a}{A}\right)^{2}\left(\frac{A}{r}\right)^{2}-e^{i 2 \psi}\left(\frac{a}{r}\right)^{2} & r>A
\end{array} .\right.
$$

We now go to a second stage focal plane by Fourier transforming Eq. (ㅁ), yielding 


$$
E_{f p 2}(\rho, \theta)=-e^{i 2 \theta} \frac{2 J_{1}(k \rho a)}{k \rho a}+e^{i 2 \theta} \frac{2 J_{1}(k \rho A)}{k \rho A}\left(\frac{a}{A}\right)^{2} .
$$

Multiplying this function by the conjugated [9] vortex $e^{-i 2 \theta}$, cancels the phase term (Fig. 2, D) leaving

$$
E_{f p 2}(\rho, \theta)=-\frac{2 J_{1}(k \rho a)}{k \rho a}+\frac{2 J_{1}(k \rho A)}{k \rho A}\left(\frac{a}{A}\right)^{2} .
$$

Transforming back to a second pupil plane, we get (Fig. $\underline{2}, \mathbf{E}^{-}$)

$$
E_{\text {pup2 }}(r, \psi)=\left\{\begin{array}{cc}
-1 & r<a \\
0 & r>a
\end{array}\right\}+\left\{\begin{array}{cc}
\left(\frac{a}{A}\right)^{2} & r<A \\
0 & r>A
\end{array}\right\} .
$$

The bright central region is then blocked by a second Lyot stop (Fig. 2, E) of radius $a$, which leaves

$$
E_{\mathrm{Lyot} 2}(r, \psi)= \begin{cases}0 & r<a \\ \left(\frac{a}{A}\right)^{2} & a<r<A \\ 0 & r>A\end{cases}
$$

The residual field distribution is thus an exact replica of the original distribution Eq. (2), but with the field reduced by $\left(\frac{a}{A}\right)^{2}$, and the intensity (per area or integrated) reduced by $\left(\frac{a}{A}\right)^{4}$ (Fig. $\left.2, \mathbf{E}^{+}\right)$. For a $10 \%$ secondary, the total residual light is then only $10^{-4}$, and the theoretical focal plane contrast at, e.g., the third Airy ring is $\sim 10^{-7}$. Thus, even with an on-axis telescope, high contrasts are achievable with such a "two-stage vortex" coronagraph. Moreover, by extension, further vortex stages likewise decrease leakage by factors of $(a / A)^{2}$, leading to a total leakage of $(a / A)^{2 n}$, where $n$ is the number of stages at the cost of additional optics and potential errors (wavefront imperfections, alignment issues, etc.). Note that multistage coronagraphy had also been proposed previously as a way to increase the working bandwidth and reduce the sensitivity to tip-tilt and stellar size leakage [10-12].

Of course secondary mirrors come with support structures (spiders). Examination of the influence of spiders on contrast is beyond the scope of this Letter, but initial calculations show that the edge enhancement property of coronagraphs and particularly optical vortices [4] allows most of the diffracted light to stay within the spiders' physical width, which can then be blocked by a Lyot stop designed to cover them at each stage. As a result, the impact of reasonably sized spiders (e.g., width $\leq 1 \%$ of the telescope diameter) is below the residual limitations imposed by the central obstruction. Thus, using a multistage vortex, high contrast can be obtained even with onaxis telescopes, important in both the space-based and ground-based cases.

This work was carried out at the Jet Propulsion Laboratory, California Institute of Technology, under contract with the National Aeronautics and Space Administration. L. Pueyo acknowledge support from the Carl Sagan Fellowship Program.

\section{References and Notes}

1. D. Mawet, P. Riaud, J. Surdej, and O. Absil, Astrophys. J. 633, 1191 (2005).

2. G. Foo, D. Palacios, and G. Swartzlander, Opt. Lett. 30, 3308 (2005).

3. C. Jenkins, MNRAS 384, 515 (2008).

4. G. Swartzlander, J. Opt. A 11, 094022 (2009).

5. D. Mawet, E. Serabyn, K. Liewer, C. Hanot, S. McEldowney, D. Shemo, and N. O'Brien, Opt. Express 17, 1902 (2009).

6. D. Mawet, J. Trauger, E. Serabyn, D. Moody, K. Liewer, J. Krist, D. Shemo, and N. O'Brien, Proc. SPIE 7440, 74400X (2009).

7. D. Mawet, E. Serabyn, K. Liewer, R. Burruss, J. Hickey, and D. Shemo, Astrophys. J. 709, 53 (2010).

8. E. Serabyn, D. Mawet, and R. Burruss, Nature 464, 1018 (2010)

9. The VVC mask applies opposite phase ramps $e^{i \phi}$ and $e^{-i \phi}$ to the orthogonal circular polarization states and also interchanges the polarization states. Thus a second VV mask downstream from the first applies the conjugated phase ramp automatically.

10. N. Yaitskova, Proc. SPIE 5905, 292 (2005).

11. L. Abe, M. Venet, K. Enya, H. Kataza, T. Nakagawa, and M. Tamura, Proc. SPIE 7014, 701467 (2008).

12. P. Baudoz, F. Assemat, R. Galicher, J. Baudrand, and A. Boccaletti, Proc. SPIE 7735, 773581 (2010). 